\title{
Evolving Graph Structures for Drug Discovery
}

\author{
Keith C.C. Chan \\ Department of Computing \\ The Hong Kong Polytechnic University \\ Hung Hom, Kowloon, Hong Kong \\ cskcchan@comp.polyu.edu.hk
}

\begin{abstract}
Computer-Aided Drug Discovery (CADD) is concerned with the use of computational techniques to determine drug structures with certain desirable properties. Evolutionary algorithms (EAs) have been proposed to evolve drug molecules by mimicking chemical reactions that cause the exchange of chemical bonds and components between molecules. For these EAs to perform their tasks, known molecular components, which can serve as building blocks for the drugs to be designed, and known chemical rules, which govern chemical combination between different components, have to be introduced before an evolutionary process can take place. To automate drug molecular design without such prior knowledge and constraints, we need a special EA that can evolve molecular graphs with minimal background knowledge. In this talk, we present one such EA that can evolves graph structures used to represent drug molecules. We show how molecular fingerprints can be used to evaluate the "fitness" of an evolved drug structure obtained at each generation during the evolutionary process. We also show how the discovering of privileged structures in many drug molecules and the use of ligand docking and binding affinity can be used as alternatives for fitness evaluating in an EA for drug design. We show how the results obtained using the proposed EA may lead to a promising approach for CADD.
\end{abstract}

